\title{
Analysis of thick, non-planar boundaries using the discontinuity analyser
}

\author{
M. W. Dunlop and T. I. Woodward \\ Space Physics Group, Physics Department, Imperial College of Science, Technology and Medicine, London, SW7 2BZ, UK \\ E-mail: m.dunlop@ic.ac.uk
}

Received: 30 November 1998 / Accepted: 12 December 1998

\begin{abstract}
The advent of missions comprised of phased arrays of spacecraft, with separation distances ranging down to at least mesoscales, provides the scientific community with an opportunity to accurately analyse the spatial and temporal dependencies of structures in space plasmas. Exploitation of the multi-point data sets, giving vastly more information than in previous missions, thereby allows unique study of their small-scale physics. It remains an outstanding problem, however, to understand in what way comparative information across spacecraft is best built into any analysis of the combined data. Different investigations appear to demand different methods of data co-ordination. Of the various multispacecraft data analysis techniques developed to affect this exploitation, the discontinuity analyser has been designed to investigate the macroscopic properties (topology and motion) of boundaries, revealed by multi-spacecraft magnetometer data, where the possibility of at least mesoscale structure is considered. It has been found that the analysis of planar structures is more straightforward than the analysis of non-planar boundaries, where the effects of topology and motion become interwoven in the data, and we argue here that it becomes necessary to customise the analysis for non-planar events to the type of structure at hand. One issue central to the discontinuity analyser, for instance, is the calculation of normal vectors to the structure. In the case of planar and 'thin' non-planar structures, the method of normal determination is welldefined, although subject to uncertainties arising from unwanted signatures. In the case of 'thick', non-planar structures, however, the method of determination becomes particularly sensitive to the type of physical sampling that is present. It is the purpose of this article to firstly review the discontinuity analyser technique and secondly, to discuss the analysis of the normals to thick non-planar structures detected in magnetometer data.
\end{abstract}

Key words. Space plasma physics (discontinuities; instruments and techniques).

\section{Introduction}

Knowledge of the motion and size of structures in space plasmas provides vital clues as to their physics (such as composition, structure, relationship to the local and/or global plasma conditions). For example, the determination of motional parameters for a non-dispersive structure permits the separation of spatial and temporal variations, which in situ produce mixed effects in time series data signatures. Single spacecraft measurements are generally insufficient for the unambiguous separation of spatial and temporal dependencies, however, except under rather extraordinary circumstances (e.g. Fairfield and Feldman, 1975). Measurements made by two or more satellites, which happen to be appropriately or fortuitously positioned, can help to unravel this mixed spatio-temporal behaviour, often through simple timing studies. Indeed several unplanned conjunctions of spacecraft, as well as planned, dual satellite missions (such as the ISEE and AMPTE missions) have resulted in numerous studies whose important conclusions hinge on establishing such knowledge of the motion of some underlying structure. For example, Greenstadt and coworkers used multi-spacecraft data sets to analyse the terrestrial bow shock and propose formation mechanisms based on the thicknesses they determined (e.g. Greenstadt et al., 1975; Morse and Greenstadt, 1976). During the ISEE-1 and -2 era, dual spacecraft data sets were again used to separate both spatial and temporal behaviour (e.g. Russell and Greenstadt, 1979; Bame et al., 1979; Russell et al., 1982; Mellott and Greenstadt, 1984). Non-planar boundary surface topology, thickness of the boundary layer, and motion has been of interest to investigators in, for example, the solar wind where interplanetary shocks have been studied (e.g. Chao and Lepping, 1974; Lepping and Chao, 1976). Another topic where such macroscopic properties have been sought are magnetopause surface waves: for example, Song et al. (1988) used simultaneous data from the closely sepa- 
rated ISEE-1 and -2 spacecraft to determine the amplitude, wavelength and period of these waves.

A major objective of the European Space Agency's four spacecraft Cluster mission is to affect this spatiotemporal separation on a routine basis and on spatial scales down to, at least, mesoscale variations. These studies used data measured during the fortuitous conjunctions of several satellites, separated by distances large compared to the typical Cluster separations (Dunlop, 1990). With the mission a reality again for launch in the year 2000, further investigation in this area is of paramount significance to the fruitful exploitation of what would be a unique data set. Clearly, such a phased array of at least four spacecraft is generally sufficient to resolve 3-D information. Nevertheless, properties of highly time dependant structures, or those which vary on spatial scales which are small compared to the array size, still present obvious problems for modelling, empirically, observed behaviour. Under certain assumptions, for example those of stationarity or comparative spatial scales, some particular analysis may be possible. But a fundamental problem always exists: how should the comparative information between spacecraft be built into any analysis? The determination of different properties demands different methods of coordination (Dunlop et al., 1988); often having a large overlap between them, with parameters in common. In terms of boundary analysis, although there is an apparent distinction between macroscopic parameters (such as motion, orientation and form) and the boundary structure itself (as sampled individually by each spacecraft), it is not always a priori obvious how spacecraft differences are to be combined with time series information, except where the boundary is planar or 'thin' (Dunlop and Woodward, 1998). Both sources of information may, in principle, provide independent knowledge of the boundary normals, for instance, but depend on these key boundary properties. The effect of different physical structure in the boundary can therefore provide a context for the time series analysis and needs to be known at some level of detail, depending upon behaviour. The general problem posed in extending the analysis to multi-spacecraft data in curved geometry is how to combine the information selfconsistently when the single spacecraft assumptions on the macroscopic behaviour are relaxed.

Dunlop and Woodward (1998) describe in detail a multi-spacecraft magnetometer data analysis technique, which they call the discontinuity analyser (DA), designed to determine the structure, orientation and motion of boundaries using calculated normal vectors at each spacecraft to the structure as well as the associated times of encounter with the structure. Their technique is focused on the use of magnetometer data only, primarily for data resolution considerations. The method is applicable to thin, non-dispersive, simple planar and non-planar structures detected by a small array of closely separated spacecraft. Their requirement of "thin" structures, where the thickness of the structure is far less than its local radius of curvature, is fundamental to the design of their method, since a unique normal direction at any point on a curved surface is only well-defined when the structure is thin. The requirement is not so critical where the boundary is planar, however. Clearly, not all structures detected in space may fit this requirement on their thickness. Nevertheless, this problem is generally present in other treatments of boundaries, and relates to the issue of how to deal with comparative information as already raised.

The purpose of this work is twofold: firstly, to summarise, in a practical way, the discontinuity analyser; and secondly, to discuss the issues for such analysis of thick, non-planar boundaries. We begin by presenting a brief but thorough description of the main facets of the DA in Sect. 2. In Sect. 2.1 we compare the quantitative information which is theoretically obtainable from the data with the information required to describe the motion and topology of simple surfaces in 1-, 2- and 3dimensions. This comparison sets the upper limit of the information that is potentially obtainable from the data and reveals possible conflicts. In Sect. 2.2 we follow this theoretical basis up with a description of the methodology underlying the two themes of the DA, namely the planar-DA and extension to the non-planar DA. We highlight the subtleties in the analysis procedures (as indicated already), in particular for the non-planar analysis which presents the more complicated problem. A pivotal concept of the non-planar technique is the degree of certainty one has in the normal vectors determined at each spacecraft. The subject of normal determination in the presence of thick boundaries is the topic of Sect. 3, where we discuss the variation of the normal determined using minimum variance analysis (Sonnerup and Cahill, 1967) for a curved, thick, 2-D model boundary under various conditions of sampling, namely data interval length, symmetry about the centre of the event, data resolution and spacecraft trajectory. A similar theme of analysis, appropriate to real spacecraft data, is presented for a barium release recorded in the magnetometer data of the AMPTE-IRM/UKS spacecraft pair in Sect. 4, confirming the model analysis. In the last section, we conclude with a discussion of the DA and the ramifications on it of the thick boundary normal analysis.

\section{Discontinuity analysis}

\subsection{Theoretical balance of information}

Consider the geometrical problem of determining the orientation, shape and motion of a non-dispersive, convecting geometrical structure in space using an array of up to four spacecraft. We assume here that the data recorded on each individual spacecraft allows us to calculate accurately the time and position at which each spacecraft encountered the structure, as well as the normal to the surface at that point. In the case of curved structures, we consider only a local expansion of the surface around the spacecraft encounter positions so that any planar curve connecting any two spacecraft positions on the surface is characterised by a single 
radius of curvature. In 2-D, for example, there is only one plane, defined by nearly co-planar normals, and we assume that the surface moves linearly with constant acceleration $a$ in that plane. We can derive equations relating the spacecraft positions, times of detection, as well as the structure's topological and motional parameters in the form:

$r_{1 j}=R \boldsymbol{n}_{j 1}-v t_{j 1}-\frac{1}{2} a t_{j 1}^{2}$

for spacecraft $1 j=2,3, \ldots$, with analogues in 3 -D (Dunlop and Woodward, 1998). Here $\boldsymbol{r}_{i j}\left(\equiv \boldsymbol{r}_{1}-\boldsymbol{r}_{j}\right.$ where $\boldsymbol{r}_{l}\left(\boldsymbol{r}_{j}\right)$ is the position vector of spacecraft $\left.1(j)\right)$ is the spatial separation of spacecraft 1 and spacecraft $j, t_{j l}$ $\left(\equiv t_{j}-t_{l}\right.$, where $t_{l}\left(t_{j}\right)$ is the time of detection of the structure at spacecraft $l(j)$ ) is the time taken for the structure to travel from spacecraft 1 to spacecraft $j$, and $\boldsymbol{n}_{j l}\left(\equiv \boldsymbol{n}_{j}-\boldsymbol{n}_{1}\right.$, where $\boldsymbol{n}_{1}\left(\operatorname{bin}_{j}\right)$ is the unit normal vector to the structure at the point where spacecraft $l(j)$ detects it) represents the deviation from planar of the structure between spacecraft $l$ and spacecraft $j$. The structure is moving with velocity $v_{1}$ when first detected (by spacecraft 1).

The motion of the surface may then be determined from the relative times of encounters combined with the geometry of the structure. In 2-D, we can consider two component vectors in the plane of the normals so that the unknowns $\left(R, v_{1}, \boldsymbol{a}\right)$ amount to five scalar quantities. We thus require four spacecraft (giving 6 equations) to determine them, or only three spacecraft if it can be assumed that there is no acceleration. Fewer spacecraft will provide insufficient data for this type of model. Table 1 below summarises the lowest order parameters of the surface which may be determined with arrays of up to four spacecraft. The plus signs refer to over constrained situations where the number of equations, in terms of the motion through the structure, is more than the number of identifiable unknowns. The negative signs refer to the reverse situation where there are less equations than unknowns (under constrained). The equals sign indicates situations where there are the same number of unknowns and equations (critically constrained).

Thus, we see that there is a rather delicate balance of the information sought on the structure versus the best possible information available from the data, and it is clear that these parameters rest on the assumptions (or

Table 1. Indication of the ability of the analysis to determine the unknowns in different physical situations and to lowest order in curvature (taken from Dunlop and Woodward, 1998)

\begin{tabular}{llllll}
\hline Dimension & $\begin{array}{l}\text { Number of } \\
\text { spacecraft: }\end{array}$ & $1 \mathrm{~s} / \mathrm{c}$ & $2 \mathrm{~s} / \mathrm{c}$ & $3 \mathrm{~s} / \mathrm{c}$ & $4 \mathrm{~s} / \mathrm{c}$ \\
\hline 1-D & No acceleration & - & $=$ & + & + \\
& Acceleration & - & - & $=$ & + \\
2-D & No acceleration & - & - & + & + \\
& Acceleration & - & - & - & + \\
$3-D$ & No acceleration & - & - & $=$ & + \\
& Acceleration & - & - & - & $=$ \\
\hline
\end{tabular}

restrictions) of motion and of topology. Moreover, any uncertainties arising from the data will offset this balance and reduce, at least quantitatively, information describing the surface. With only four spacecraft, it is not possible to demonstrate the absence of acceleration, if some general curvature is present. Since the acceleration and curvature terms can enter into Eq. (1) at the same order, not even qualitative information can be inferred. Conversely, however, if the normals indicate the structure is planar, a full motional analysis can be performed without assumption, as we outline in Sect. 2.2. It is worth noting here, in view of the later discussion, that for planar boundaries, which are assumed to have only constant velocity, the common normal to the boundary can be determined from the timing and separation information (Russell et al., 1983). The question which arises is what interpretation should be given to any differences between this normal and those determined individually. In fact these could arise from a number of sources: actual acceleration, data errors, boundary properties, and so on, so procedure is then not clear.

\subsection{The discontinuity analyser}

The analysis of the motion of time stationary structures, which are demonstrably planar, has been discussed at length in Dunlop and Woodward (1998) and was termed the planar discontinuity analyser (planar-DA). We refer the interested reader to that article for the details. The discussion here assumes that we have magnetometer data, detected simultaneously on two or more spacecraft, which reveals similar data signatures of magnetic structure from which arrival times may be estimated. This requires that we are dealing with 'thin' boundaries (as defined in Dunlop and Woodward, 1998): the thickness of the boundary is small in comparison to its local radius of curvature, and that the spacecraft separations are well in excess of the thickness of the structure. Additionally, the quality of any estimates of the macroscopic quantities depends upon the detailed microstructure in the sampled discontinuity, such as the presence of natural noise, wave or other properties, which can confuse simple model assumptions for the boundary unless either the structure is sampled fortuitously or judicious spectral filtering is possible. The latter will depend upon the degree to which the properties are in some sense conflicting in their effect (see Dunlop et al., 1995; Dunlop et al., 1996, for more discussion of this point in the context of multi-spacecraft measurements).

The methodology on which the planar-DA is designed consists of four principle stages (Fig. 1): data preselection, normal stability, crossing times and combined analysis of normals. The result estimates the motional parameters in the case of a planar structure. Because this procedure, when applicable, first establishes individual boundary normals at each crossing, the motional analysis can be made as an explicit fit to the apparent motion; complex motion simply degrades the fit. No 


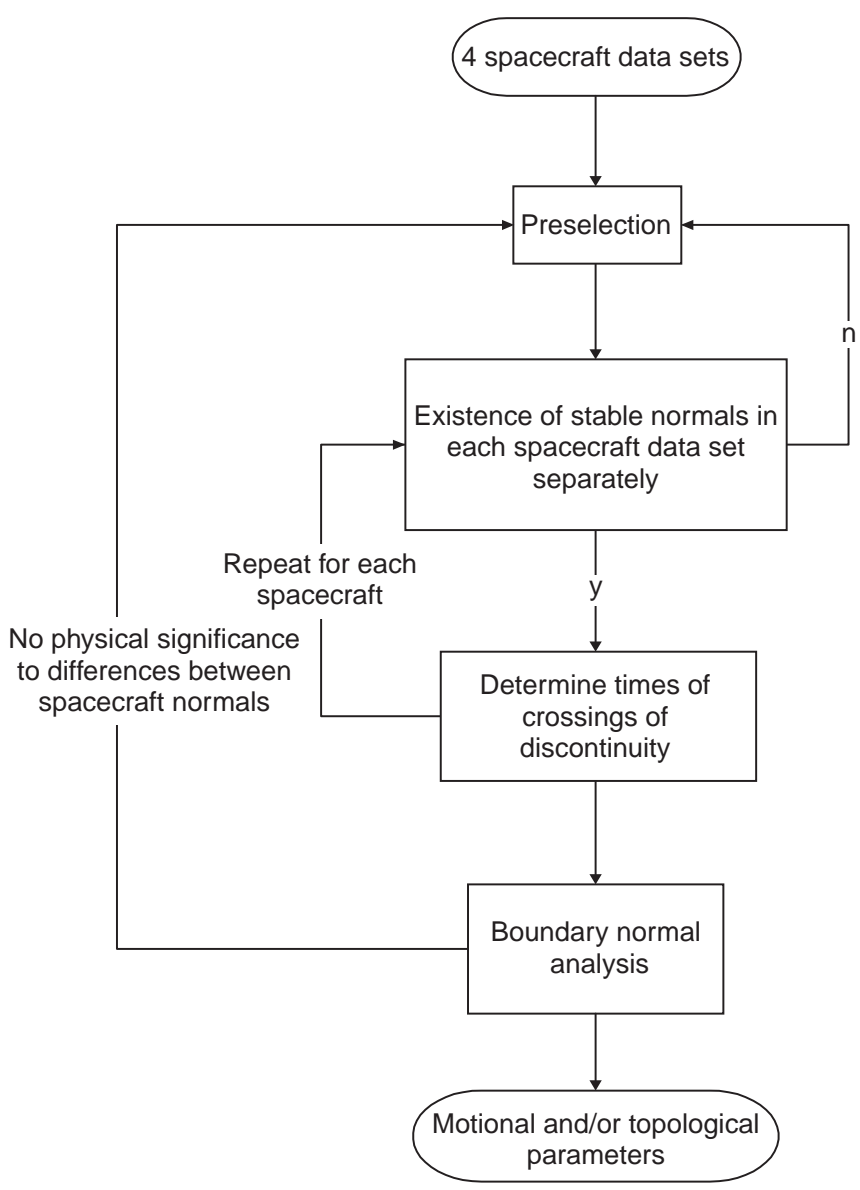

Fig. 1. Conceptual flow chart for the boundary analysis, taken from Dunlop and Woodward (1998)

assumptions are made regarding the parameters determined so that the method is essentially self-consistent, within the limits of applicability. The description in Dunlop and Woodward (1998), therefore, includes data pre-selection and normal stability (as well as consistency) issues which control its applicability.

In the data pre-selection process, candidate data intervals which may possess a structure signature detected on two or more spacecraft are identified. The next process of the algorithm aims at determining the normals to the structure at each spacecraft where the signature is detected from single spacecraft measurements. Techniques such as minimum variance analysis (Sonnerup and Cahill, 1967) are employed on a set of nested data intervals to assess the stability of any determined normal. Should a failure to detect a stable normal at any spacecraft arise, reconsideration of that data interval in terms of further pre-selection or prefiltering is carried out via the data pre-selection process. On the other hand, if suitable normals are found, the next step is to measure the times at which each encounter with the respective spacecraft occurred. This may be done by curve fitting to the data signature or otherwise (e.g. by inspection). Finally having ascertained the existence, if any, of suitable normals to the structure as well as their associated crossing times, the information is now combined across the spacecraft and a normal analysis is carried out.

If the normals determined at each spacecraft are parallel and there are three or more spacecraft available, then the boundary is planar. That is, when the deviations $\delta \mathbf{n}_{\alpha}$ of each spacecraft $\alpha$ s normal $\mathbf{n}_{\alpha}$ from the average normal, $\langle\mathbf{n}\rangle \equiv \sum \mathbf{n}_{\alpha} /\left|\sum \mathbf{n}_{\alpha}\right|$ (calculated in the first box in Fig. 2), are small, strong planarity exists and we may proceed to compute the motion of the structure. This is done by fitting a linear or quadratic curve to points with co-ordinates corresponding to the relative displacements, $\quad \Delta r_{\|_{\alpha}} \equiv r_{\|_{\alpha}}-r_{\| 1} \quad$ (where $\quad r_{\|_{\alpha}} \equiv \mathbf{r}_{\alpha} \cdot\langle\mathbf{n}\rangle$ ), between the spacecraft parallel to the average normal to the structure and the associated relative transit times $\Delta t_{\alpha} \equiv t_{\alpha}-t_{l}$, according to the level of noise in the signature. Thus we may determine the parameters in the following equation of motion for the planar structure

$\Delta r=\mathrm{v}_{\|}^{\prime} \Delta t+\frac{1}{2} a_{\|} \Delta t^{2}$

where $\mathrm{v}_{\|}^{1}$ and $a_{\|}$are the velocity of initial detection and acceleration of the structure parallel to $\langle\mathbf{n}\rangle$. This is the planar-DA technique and the equation is the analogue of Eq. (1) for planar structures. It is a fairly simple matter to compare the significance in errors arising from timing, position, or uncertainties in the normals, to the acceleration term and it is clear that for a wide variety of event characteristics, the method can tolerate up to $10 \%$ errors in the individual ns.

Figure 2 also indicates the case of non-parallel normals (for which $\delta \mathbf{n}_{\alpha}>10 \%$ ), which, of course falls into the realm of non-planar analysis and is somewhat more complicated than its planar counterpart. The existence of stable, distinctly non-parallel normals, however, is not necessarily an indication of curvature and this is addressed in the box labelled "significance of $\delta \mathbf{n}_{\alpha}$ " in Fig. 2. By "physical" significance we mean the relevance to a planar discontinuity analysis. For example, the $\delta \mathbf{n}_{\alpha}$ may arise from effects other than simple boundary structure, such as surface waves. If the origin of the normal differences is believed to be a curved thin magnetic structure, then we can attempt to characterise the surface and its motion more fully. Systematic differences indicate the probable existence of curved field geometry and we can attempt a quantitative analysis of the surface curvature and motion. Conversely, if the normals show differences that are inconsistent with simple curved field geometry but that are, nevertheless, believed to fundamentally derive from curved field geometry, then application of a planar analysis provides a less quantitative estimate of the velocity (i.e. an estimate representing only a mean velocity) and a qualitative indication of nonplanarity. Thus, the planar-DA as applied, can always qualitatively predict the existence of a curved boundary. Limits on the quantities determined are indicated in Fig. 2. Calculating an acceleration term in the presence of such large deviations in the normals is essentially meaningless. Furthermore, curvature can bias the estimate of velocity and even masquerade as an 


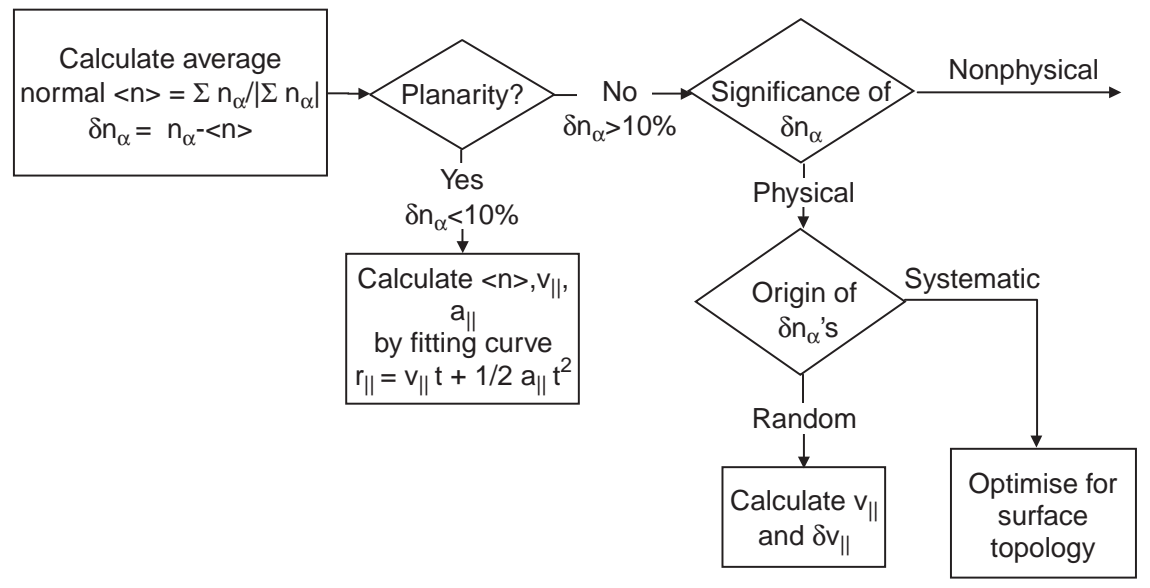

Fig. 2. The boundary normal analysis flowchart, taken from Dunlop and Woodward (1998) apparent acceleration. Dunlop and Woodward (1998) illustrate how this occurs for the planar-DA and one can easily generalise this reasoning to the non-planar case. Thus motional and topological properties of a surface are interwoven in their representation in the data. Consequently any analysis making an assumption on the one affects the other.

As indicated, once the analysis demonstrates the presence of curvature, we can attempt to determine the topology and motion of the surface. There are two routes we can pursue: namely a discrete sequential analysis, or a combined self-consistent analysis. Clearly, these two approaches to the analysis of non-planar surfaces must be constructed on different methodologies. The latter route entails the calculation of the normals, surface motion and topology simultaneously (see later) and is in contrast to the former route, which involves first determining the normals to the surface at each spacecraft crossing point, secondly assuming some motional as well as topological form for the surface, and finally calculating those topological and motional parameters which characterise the surface. This is essentially the methodology used in structuring the planar-DA technique and as treated theoretically in Sect. 2.1. Independent determination of the boundary normals clearly provides a direct test of planarity, which then sets the context for the following motional analysis. With magnetic field data alone, the converse of this is not possible, i.e. there is no equivalent test for events with constant velocity. Motional information therefore has to be either assumed, or obtained from other instrument data (e.g. plasma velocity, or DeHoffmannTeller analysis, Khrabrov and Sonnerup, 1998).

Given some knowledge or assumption of motion, the crossings at each spacecraft can in principle (Sect. 2.1) be mapped back to the positions at the time of the first crossing. Then using the normals determined at each spacecraft position, a surface may be fitted to the mapped points, with the quantities describing the motion (i.e. velocity and acceleration) treated as parameters in the fit. In Table 1, however, we note that for the cases that are under-constrained, qualitative indications may still be obtained, such as testing the existence of curvature, or acceleration. Alternatively, quantitative analysis can be attempted in principle, given certain assumptions of the macroscopic properties. We can assume the motion has a constant velocity for the purpose of pursuing a curvature analysis, for instance. Clearly, from the table, this appears to allow lowest order determination of curvature to be made in more circumstances (up to 3-D, or with less spacecraft). For such an assumption of constant motion the discrete analysis can be pursued in the manner discussed by Mottez and Chanteur (1994). However, the acceleration and curvature terms in Eq. (1) enter at least at the same order. Thus, the surface topology is particularly sensitive to deviations from this motional assumption for an actual event.

It is, therefore, never known whether the curvature analysis is fundamentally in error (i.e. an artifact) as a result of an acceleration in the motion which had not been taken into account. The converse situation occurs if planarity is assumed, anticipating an analysis such as the planar-DA method. The presence of actual curvature affects the motional fit at the same order as the acceleration term, and would infer an artificial acceleration. With a minimum of four spacecraft and to lowest order in curvature, the motion can be determined, without assumptions, up to a constant acceleration term, given that the normals can be estimated by some independent method. In this case, therefore, the result of lowest order determination of the unknowns will have some uncertainties, but is in principle, self-consistent. A more complex surface or motion is just represented approximately by the lowest order terms, but not anomalously.

\section{Thick, non-planar boundaries}

In the presence of curved field geometry, normal determination is a subtle issue, particularly when the boundary is not thin. Systematic uncertainties on the normals arise, and these disrupt the delicate balance of information indicated in Table 1. This then questions the validity of performing a surface analysis, since uncertainties in the normals imply uncertainties in both the topology and the acceleration of the structure. In 
contrast to the discrete methodology, Dunlop and Woodward (1998) introduced the concept of the combined approach which recognises that independent determination of the normals can be compromised. This methodology is based on the fully self-consistent determination of the surface topology and motion of the structure past the spacecraft array, cast in the framework of an inversion problem. They also discussed a compromise methodology between that of the discrete analysis and that of the combined approach. In this they suggested the explicit use of single-spacecraft determined normals weighted by confidence factors. In the following section we discuss normal determination in the presence of thick, non-planar boundaries, first presenting the conceptual scenario and then illustrating our ideas with an analysis of a model 2-D structure. This is followed up in Sect. 4 with the analysis of an event recorded in the dual spacecraft AMPTE-IRM/UKS mission.

\subsection{Normal determination}

In single spacecraft analysis, normal determination is affected by the direction of motion through the boundary of the spacecraft (particularly if the curvature is high) as well as the nature of the data interval used in the normal analysis (Fig. 3). Strong curvature arises when the thickness of the boundary surface is not negligible in comparison to the radius of curvature of the surface. In this case normal determination becomes difficult, as a result of the need to identify a finite data interval (which maps to a distance along the motion) through the boundary in order to perform a boundary normal analysis. Unless this distance is small on the scale of the curvature, the field structure will not wholly represent the boundary orientation local to the crossing, thus affecting the implied normal direction (as indicated in Fig. 3). Boundary normal estimates will produce some average normal which will be sensitive to the interval chosen.

The individual normals are therefore dependent on the spacecraft trajectory, with the dependence being related to the a priori unknown form of the surface. Any result of surface fitting cannot therefore be fully decoupled from the determination of normals. This raises the question of whether or not a full curvature analysis can be done quantitatively unless curvature is not significant on the scale of the boundary layer. Nevertheless, normals determined at two spacecraft, which deviate by a direction well in excess of that implied by the uncertainties, do indicate curved field geometry. For real events, however, it is not known, a priori, what weighting should be given to the errors in the individual normals, in order to then apply some optimisation of the surface fit. One can either attempt to refine the uncertainties using the implied curvature (iterative process) and proceed with the discrete analysis, or feed the data into the combined methodology, knowing that real curvature exists. We explore the applicability of the former route next.

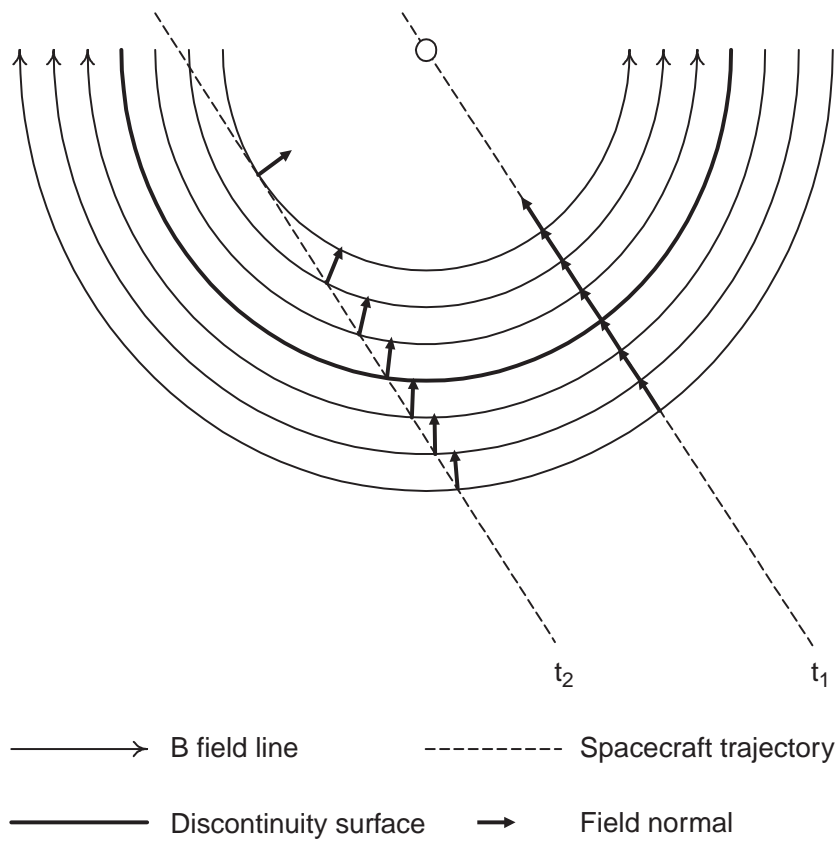

Fig. 3. Diagrammatic illustration of the effect of thick, curved boundaries on normal determination. Shown is a circular discontinuity (thick curve), with two spacecraft trajectories $\left(t_{1}\right.$ and $\left.t_{2}\right)$ and normal vectors (short thick arrows) to the field lines (thin curves with arrowheads indicating field direction) along each trajectory, taken from Dunlop and Woodward (1998)

\subsection{Data analysis}

In order to quantitatively illustrate the variation of the normal along sampling trajectories through curved boundaries, we make use of a simple 2-D model, performing several fly-throughs or sampling trajectories and analyse the data thus recorded in various ways. The 2-D model we use is shown in the upper panel of Fig. 4, together with four sampling trajectories labelled $t_{\mathrm{x}}=0,1,2,8$. In the lower panel, typical magnetic field data signatures for the samplings are indicated.

We highlight the effects of data interval length, symmetry about the centre of the boundary, data resolution and spacecraft trajectory on the calculation of boundary normals using MVA (hereafter referred to as MVA normals). Since we have defined a simple model, theoretical normals are readily calculated in order to assess the accuracy of the MVA normals. Similar analyses have also been carried out with other curved 2-D models, obtaining qualitatively similar results.

Figure 4 illustrates the relative positions of four sampling trajectories across a 2-D circular discontinuity surface with a radius of curvature of 10 units (Fig. 4a), as well as a typical data signature (Fig. 4b). The first trajectory $t_{\mathrm{x}=0}$ traverses along the $y$-axis, while the others, $t_{\mathrm{x}=1,2,8}$, traverse parallel to the $y$-axis along the appropriate $x$-value. We shall compare and contrast the described effects on the MVA normals using these four trajectories. Firstly, consider the four trajectories in relation to the point symmetry of this particular model. Trajectory $t_{\mathrm{x}=0}$ passes along a radial direction, while the 
other trajectories are increasingly inclined to this characteristic direction. Three characteristic runs were performed: one, where the data interval used is nested about the crossing, successively increasing its size; one, where the interval is shifted relative to the crossing time, successively changing the ratio of data intervals before and after the crossing, and another, where the effects of data resolution were explored.
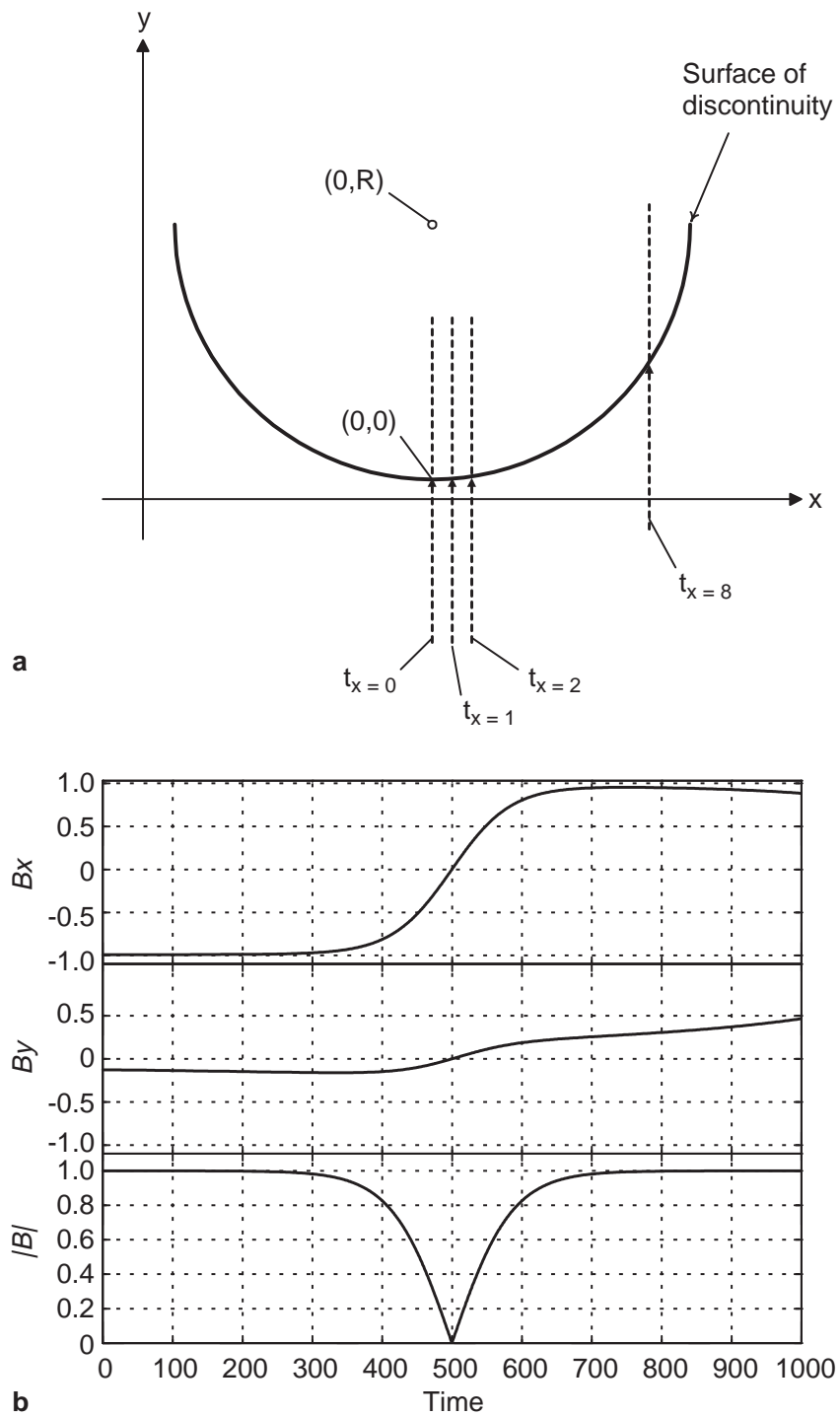

Fig. 4a, b. Schematic drawing of sampling trajectories through a circular discontinuity model and the associated data (time and magnetic field values in arbitrary units). The magnetic field model is defined as follows:

$b=\nabla \varphi \times \hat{z} ; \varphi \equiv B_{0 l} \log \left[2 \cosh \left(\frac{g(x, y)}{\sigma}\right)\right] ;$

$g(x, y) \equiv R-\sqrt{\left(x^{2}+(y-R)^{2}\right)} ; y<R$

where $2 \sigma$ is the boundary thickness, $B_{01}$ is a constant with units of length times magnetic field and $R$ is the radius of curvature of the discontinuity surface. The field line topology is indicated in Fig. 3. In this model the strength of the field decreases to zero on approaching the boundary from one side, before flipping direction and increasing its strength on the other side
Figure 5, which is a plot of the angular deviation of the MVA normals from the theoretical ones versus the relative length of the data interval over which the MVA normal is calculated, illustrates the effect of nesting. Note that we do not calculate the sense, clockwise or anti-clockwise, of the angular deviations. It is seen that the effect is more marked the greater the inclination of the trajectory is to the characteristic direction, is greater for longer data intervals, and can be of the order of several degrees. As the interval approaches a thin sheath around the centre of the discontinuity, or if $t_{\mathrm{x}=0}$ is chosen, the effect is minimal. This is because for longer and more inclined trajectories the spacecraft samples magnetic fields of very different directions over the analysis interval, and hence of different normal directions. However, there is a slight anomaly from zero deviation for all trajectories, except $t_{\mathrm{x}=0}$, at the smallest data interval, as well as a minimum deviation which is not at the shortest interval. This is explained when we consider the asymmetry of the data interval about the discontinuity centre.

In order to study the effect of data interval asymmetry, we sample along all the trajectories, $t_{\mathrm{x}=0,1,2,8}$, with some resolution (e.g. 1000 points for the whole data interval). Then taking a fixed length of data (e.g. 640 points) shorter than the whole interval sampled and containing the centre of the discontinuity somewhere inside, we determine the asymmetry of the chosen subinterval and perform MVA on it. This is repeated for several asymmetries and for each trajectory. We define an asymmetry index of the selected data interval to be the ratio of the number of data points recorded before the centre of the discontinuity is encountered to that measured after encounter, with all these points within in the chosen interval length (e.g. 640 points). Thus symmetry indices can only be positive. Unit symmetry index implies that there are an equal number of points on either side of the discontinuity centre: i.e. a perfectly symmetric interval.

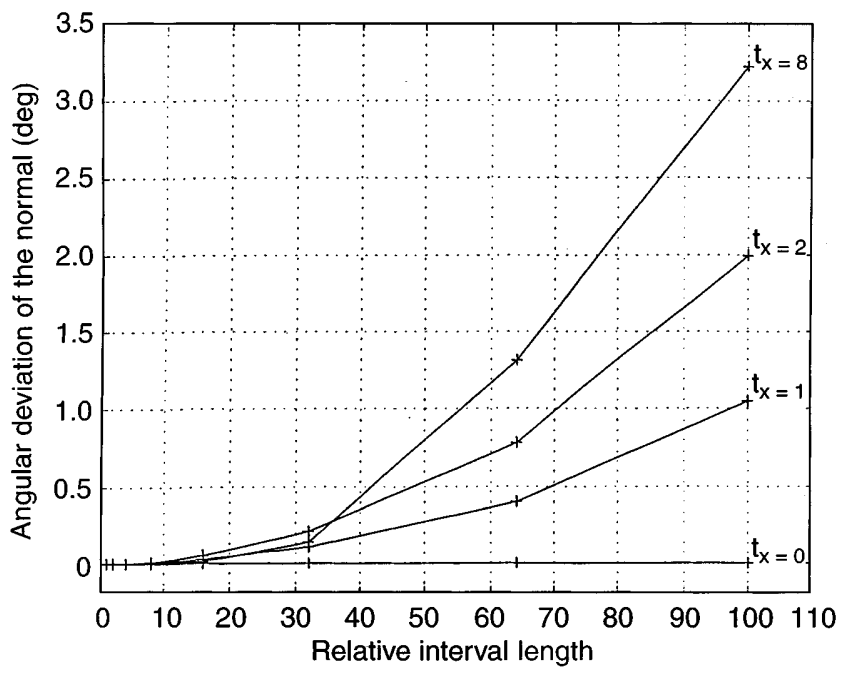

Fig. 5. Sensitivity of MVA analysis to the model shown in Figure 5 in terms of interval nesting. 
Figure 6 shows the dependence of the deviation of the MVA normal with data interval symmetry index. Again, the effect is greater the higher the inclination of the trajectory. For highly inclined trajectories, there is more than an order of magnitude change in the deviations of the MVA normals as the asymmetry of the data interval is varied. Furthermore, the minimum deviations occur for non-unitary symmetry indices (i.e. asymmetric data intervals) and this minimum varies according to the inclination of the trajectory. The minimum occurs at higher symmetry indices (i.e. more asymmetric data intervals) for lower trajectory inclinations. Since the curvature of the field lines is tighter after the discontinuity centre is encountered compared to the situation prior to encounter, the corresponding field normal directions are (monotonically) changing faster after rather than before encounter. Since MVA finds some average normal over the sampled data interval, you would not expect to see zero deviation for a perfectly symmetric interval, and moreover would expect minimal/zero deviation to occur for symmetry indices greater than unity.

The effects of data resolution on the calculation of discontinuity normals is illustrated in Fig. 7, using one analysis. It shows that the deviation is of the order of a few degrees. Intuitively we expect to find that MVA normals are of higher accuracy the greater the data resolution, as in Fig. 7. However, this is not always the case and the situation is not as clear-cut as the effects described. We leave this to a more detailed later study. The main point we wish to make here is that data resolution does have an affect on MVA normal determination and is of the order of a few degrees.

It is not surprising, of course, that MVA provides normal estimates in curved geometry which deviate from the geometric normals indicated by the models. We explore the effect here to investigate the quantitative biasing on the normals in the sense of their use within the discrete methodology described; i.e. as a single

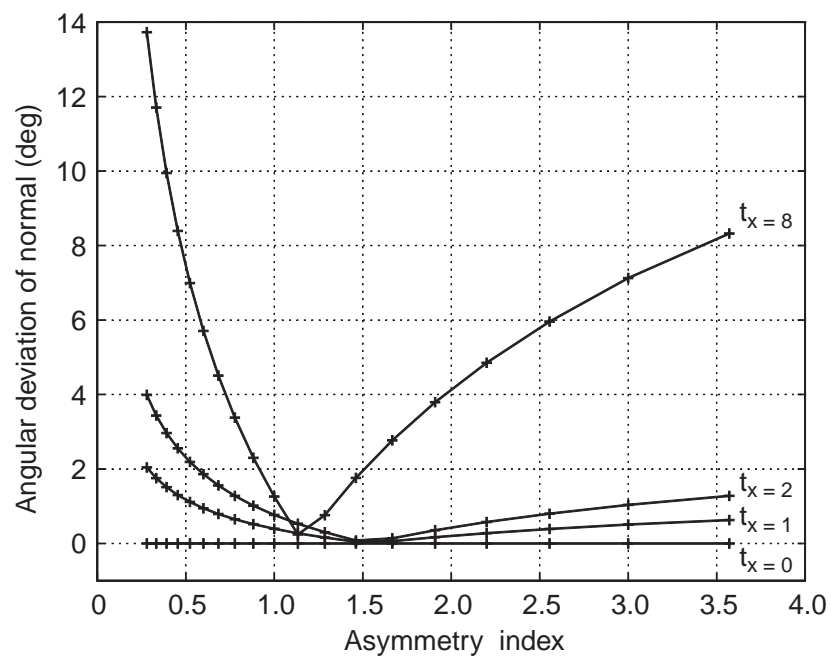

Fig. 6. Sensitivity of MVA analysis to the model shown in Figure 5 in terms of interval asymmetry. spacecraft analysis for the surface normal $n$, feeding into the equation of motion. This systematic error depends on the model used, of course, but the trends show that the significance of this error with respect to the overall deviations of the normals between spacecraft can be optimised by choice of trajectory, given the data sampling achieved. The sampling depends upon the combination of the minimum nesting interval applied and the data resolution with respect to the characteristic spatial scale of the structure. In practice, the sampling achieved depends upon the thin boundary properties of an event. The trajectory selected then sets the spacecraft separation scale with respect to the characteristic scale of the model.

Clearly, different events will be sampled in different ways in situ so that for some the systematic error will be comparatively large and for others comparatively small. In the latter case, the discrete methodology may be used for such selected events to estimate both lowest order curvature and acceleration terms. As indicated by the flow in Fig. 2, the rejection of qualitatively unsuitable events may be done if the normal analysis shows unstable results (with respect to nesting or shifted intervals) in the presence of significant deviations between the spacecraft normals.

\section{Analysis of a real event}

Figure 8 shows a real event measured by the combined AMPTE-IRM (Fig. 8a) and UKS (Fig. 8b) spacecraft during a solar wind barium release (see papers in Nature, 320, 700-726, 1986), plotted in GSE co-ordinates. Both spacecraft were thought to have sampled an intense plasma cloud boundary at about the time (within a few seconds) indicated by the solid vertical lines on the $|B|$ plots in the upper panels. Because of cloud expansion following the release, this boundary should be strongly curved between the two spacecraft and this appears to

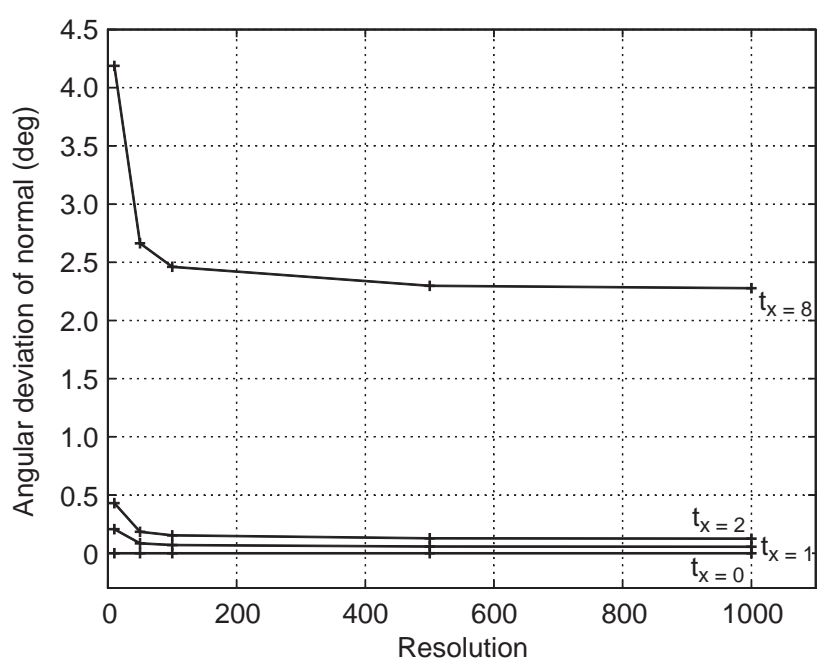

Fig. 7. Sensitivity of MVA analysis to the model shown in Fig. 5 in terms of data resolution 
be confirmed by boundary normals obtained from each data set. The sense of the curvature implied by the normals, which were obtained using a standard run, is described in Dunlop et al. (1996) and is consistent with an expanding cloud where the effective tilt between the normal directions is $\sim 30-40^{\circ}$. Clearly, this event would be unsuitable for the discrete analysis in the manner discussed in Sect. 3.2.

We therefore expect that MVA will return a biased normal estimate, which will be sensitive to the actual sampling path of the spacecraft, as discussed. To investi- gate the sensitivity of the MVA normals, a similar set of runs has been performed, successively adjusting the data intervals used to determine them. For this real event, covered only by two spacecraft, we clearly only have two fixed trajectories, and have fixed data resolution. We do not have the equivalent of Fig. 7, therefore, but can analyse in the spirit of Figs. 5 and 6. Effectively, the IRM spacecraft corresponds to the model sampling trajectory, $t_{\mathrm{x}=0}$, being in the centre of the cloud. The UKS spacecraft corresponds to an intermediate trajectory, inclined at $\sim 30-40^{\circ}$ to the boundary, traversing the outer cloud
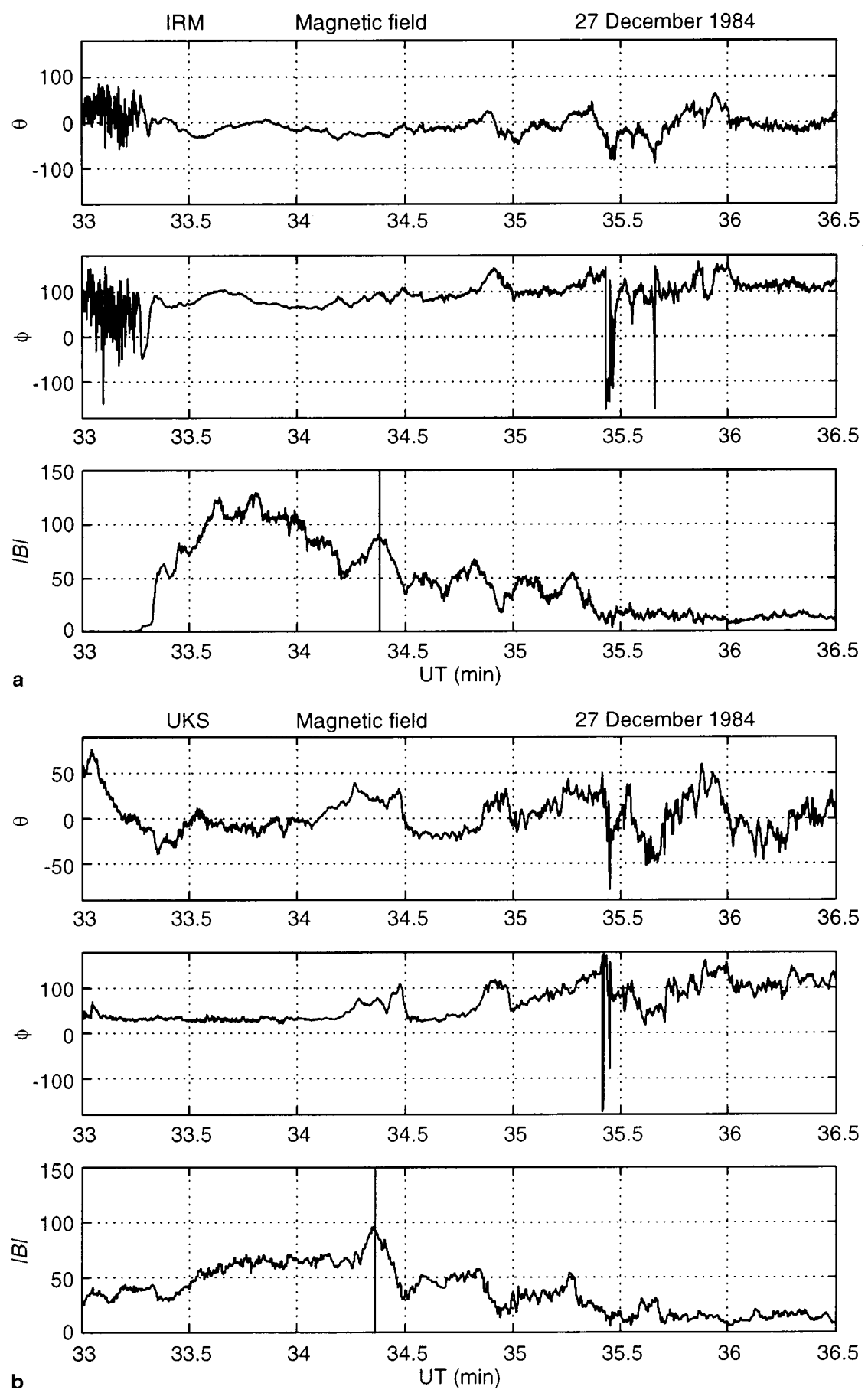

Fig. 8. Example of a curved discontinuity from the AMPTE-UKS/IRM solar wind barium release, as discussed in the text 
region before exiting. The data signature, however, is complicated by properties other than the simple model described. In particular, the magnetic signature changes character over the extent of the cloud, and between the two spacecraft (since they traverse different regions). Also, upstream conditions (times following the exit time) are obviously not static and contain clear wave power at characteristic frequencies. Thus, we cannot test the quantitative comparison between the trajectories (spacecraft), but Fig. 9 does show the sensitivity to changes in the MVA interval extremely well.

The two vertical panels in Fig. 9a show the equivalent trends to those in Fig. 5 for the two spacecraft separately, where the analysis interval has been increased in steps, from $20 \mathrm{~s}$ up to one and a half minutes, centred on 12:34:20 UT. Both panels show initially, the steady increase in deviation of the boundary normal, here defined as the angle of successive estimates with respect to the inner interval. At some point the trend breaks down, since other properties are being sampled well within or upstream of the cloud and its structure is likely to be still changing in time. The panels in Fig. 9b are perhaps more significant, since they show the equivalent trends to those in Fig. 6, where an analysis interval of $2 \mathrm{~min}$ (for UKS), and $1 \mathrm{~min}$ (for IRM), has been shifted in steps of $10 \mathrm{~s}$ about 12:34:20 UT. The interval asymmetry has been defined as the ratio of inner to outer portions of the time interval, as for Fig. 6. Clearly, both spacecraft, but particularly UKS, show deviations (defined with respect to the symmetric interval) which have closely similar character to those in Fig. 6.

\section{Discussion and conclusion}

We have presented a summary of the DA technique, based on magnetometer data. This seeks to characterise the topological structure and motion of thin, nondispersive structures present in space magneto-plasmas. It assumes in situ detection by a small array (up to four) of closely separated spacecraft. The methodology underlying the technique here, namely the discrete, sequential approach, first determines topological parameters, defined with respect to boundary normals, independently before these are fed into the motional equations. The planar form of the DA, which is based on the discrete analysis framework, relies on the independent determination of boundary normals to give a direct demonstration of planarity, and this then sets the context for the subsequent motional analysis. We point out that in the case of non-planar structures, topological and motional properties compete for: (1) their representation in the data, and (2) the nature of the analysis that can be performed. Techniques such as those presented by Chao and Lepping (1974) and Russell et al. (1983) rely on an assumption of planarity, rather than its demonstration. Mottez and Chanteur (1994) assume constant velocity and relax the planarity assumption, relying on accurate single spacecraft-determined normal directions, in their analysis. Thus, these techniques are only mutually consistent with their assumptions; but these assumptions may not be consis- tent with the data, leading to the determination of an anomalous motion, on the one hand, or an anomalous curvature on the other.

The non-planar form of the DA therefore represents the more intricate analysis problem, with assumptions on topology affecting the motional determination and vice versa. The discrete methodology orders the analysis sequentially, but under the requirement that boundary normals can be accurately obtained (from individual, time series analysis). We can ask, however, how the individual normal directions are adjusted to take account of a surface fitting procedure: this implies that equal weight is given to each. Uncertainties arising from normal determinations in particular, can therefore be a major contributor to the procedural dilemma for establishing all macroscopic parameters, since it is not obvious that normal directions, so determined, are unbiased in curved geometry. Errors are likely to be systematic, especially in the context of boundaries that are not thin. A combined approach has been outlined by Dunlop and Woodward (1998), which attempts to deal with this dilemma, in which all surface and motional parameters are determined simultaneously: some weighted adjustment of the surface away from the strict orientations of the boundary normals is allowed in order to optimise for all parameters. Nevertheless, a data regime is applicable for the DA technique, as conceived, in which boundary structure can be described as approximately thin.

In view of the importance of this issue, we have presented an analysis of the variation of normals (determined using minimum variance analysis) to a curved 2-D model boundary from actual model normals under various scenarios of sampling. The model topologically orders the field far from its centre and is hence 'thick'. We found that the greatest deviations arose for asymmetry of the analysis data interval about the centre of the event, with higher deviations occurring for trajectories highly inclined to the characteristic directions of the model. Smaller deviations were observed for the size of the data interval and data resolution, and again the greater the inclination of the sampling trajectory to the characteristic directions of the model the greater the deviations were in both cases. Analysis of barium release data from the AMPTE-IRM/UKS spacecraft provided a real event demonstration of the trends found with the model event, indicating significant deviations of the normals from the average normal of up to $90^{\circ}$, and confirming the model analysis. Thus, we conclude that normal determination in curved geometries is open to error, and furthermore cannot be completely de-coupled from the determination of the surface topology. One could attempt to refine the uncertainties using the implied curvature (iterative process) and proceed with the discrete analysis methodology. Conversely the combined methodology may be adopted, using confidence factors implied by analysis similar to that described here. Furthermore, a possible event selection test, to identify 'thin' boundaries by combining the individual time series analyses, has been conjectured by Dunlop and Woodward (1998). 

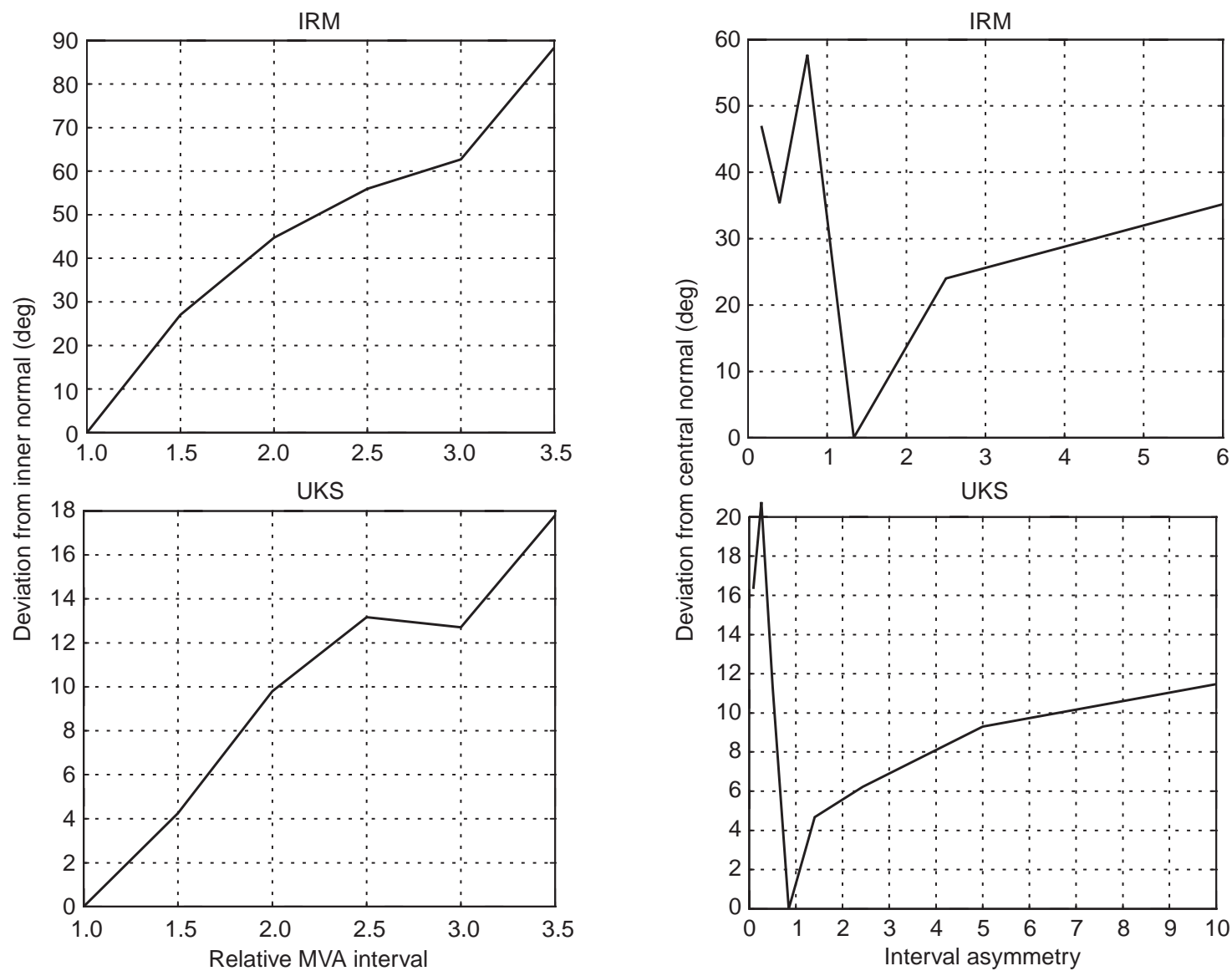

Fig. 9a, b. Sensitivity of MVA analysis to the event shown in Fig. 9., a in terms of nesting and $\mathbf{b}$ in terms of asymmetry

In any multi-spacecraft analysis, it is generally important to understand in what way comparative information across spacecraft is best built into any analysis of the combined data. Although for the DA, there is at first sight an apparently clear separation of the handling of multi-point and time series information, in general, different investigations appear to demand different methods of data co-ordination. The use of other instrument data, of course, can potentially add information on structure, such as independent determination of boundary motion in the case of electric field measurements, or via DeHoffmann-Teller analysis using plasma data. This must be carefully assessed, however, since with data restrictions on resolution and quality of these other data sets, a different analysis methodology may required and such additional measurements are most easily added as independent information on the event. The same is true of analysis using more than four spacecraft (the minimum for direct 3-D spatial information), where there will be at least a play off between use of the redundant information to improve spatial accuracy or establish temporal evolution.

Acknowledgements. We would like to thank Professor Karl-Heinz Glassmeier and Professor Peter Weidelt of the Institut für Geophysik und Meteorologie, Technische Universität Braunschweig, Germany for useful discussions on elements of this work. We acknowledge financial support from PPARC in the U.K.

\section{References}

Bame, S. J., J. R. Asbridge, J. T. Gosling, M. Halbig, G. Paschmann, N. Sckopke, and H. Rosenbauer, High temporal resolution observations of electron heating at the bow shock, Space Sci. Rev., 23, 75, 1979.

Chao, J. K., and R. P. Lepping, A correlative study of ssc'c, interplanetary shocks, and solar activity, J. Geophys. Res., 79, 1799-1807, 1974.

Dunlop M. W., Review of the Cluster orbit and separation strategy: consequence for measurements, SP-306, p17, ESA Publications, Noordwijk, The Netherlands, February, 1990.

Dunlop, M. W., and T. I. Woodward, Discontinuity analysis: orientation and motion, in Analysis methods for multispacecraft data, ISSI Scientific Report SR-001, p271, ESA Publications, Noordwijk, The Netherlands, 1998.

Dunlop, M. W., D. J. Southwood, K.-H. Glassmeier, and F. M. Neubauer, Analysis of multipoint magnetometer data, $A d v$. Space Res., 8, (9)273-(9)277, 1988.

Dunlop, M. W., T. I. Woodward, and C. J. Farrugia, Minimum variance analysis: CLUSTER themes, Proc. Cluster workshop on Physical Measurements and Mission Orientated Theory, ESA SP-371, 33-42, 1995.

Dunlop, M. W., T. I. Woodward, U. Motschmann, D. J. Southwood, and A. Balogh, Analysis of non-planar structures with multipoint measurements, Adv. Space Res., 18 (8),309, 1996.

Fairfield, D. H., and W. C. Feldman, Standing waves at low Mach number laminar bow shocks, J. Geophys. Res., 80, 515-522, 1975.

Greenstadt, E. W., C. T. Russell, F. L. Scarf, V. Formisano, and M. Neugebauer, Structure of the quasi-perpendicular laminar bow shock, J. Geophys. Res., 80, 502-514, 1975. 
Khrabrov, A. V., and B. U. Ö. Sonnerup, DeHoffmann-Teller analysis, in Analysis methods for multispacecraft data, ISSI Scientific Report SR-001, p221, ESA Publications, Noordwijk, The Netherlands, 1998.

Lepping, R. P., and J. K. Chao, A shock surface geometry: the February 15-16, 1967, event, J. Geophys. Res., 81, 60-64, 1976.

Mellott, M. M., and E. W. Greenstadt, The structure of oblique subcritical bow shocks: ISEE-1 and -2 observations, J. Geophys. Res, 89, 2151, 1984.

Morse D. L., and E. W. Greenstadt, Thickness of magnetic structures associated with the Earth's bow shock, J. Geophys. Res., 81, 1791, 1976.

Mottez, F., and G. Chanteur, Surface crossing by a group of satellites; a theoretical study, J. Geophys. Res., 99, 13,49913,507, 1994.
Russell, C. T., and E. W. Greenstadt, Initial ISEE magnetometer results: shock observations, Space Sci. Rev., 3-37, 1979

Russell, C. T., M. M. Hoppe, W. A. Livesey, J. T. Gosling, and S. J. Bame, ISEE-1 and -2 observations of laminar bow shocks: Velocity and thickness, Geophys. Res. Lett., 9, 1171, 1982.

Russell, C. T., M. M. Mellott, E. J. Smith, and J. H. King, Multiple observations of interplanetary shocks: four spacecraft determination of shock normals, J. Geophys. Res., 88, 4739-4748, 1983.

Song, P., R. C. Elphic, and C. T. Russell, Multi-spacecraft observations of magnetopause surface waves: ISEE-1 and -2 determinations of amplitude, wavelength and period, $A d v$. Space Res., 8, (9)245-(9)248, 1988.

Sonnerup, B. U. Ö., and L. J. Cahill, Magnetopause structure and attitude from Explorer 12 observations, J. Geophys. Res., 72, 171-183, 1967. 